\title{
Applying Super-Resolution to Panoramic Mosaics
}

\author{
Assaf Zomet Shmuel Peleg \\ Institute of Computer Science \\ The Hebrew University of Jerusalem \\ 91904 Jerusalem, Israel \\ \{zomet, peleg\}@cs.huji.ac.il
}

\begin{abstract}
Mosaicing and super resolution are two ways to combine information from multiple frames in video sequences. Mosaicing displays the information of multiple frames in a single panoramic image. Super-resolution uses regions which appear in multiple frames to improve resolution and reduce noise. Simple combination of the two methods is problematic since the alignment used for mosaicing may not be accurate enough over the entire region for super resolution. In this paper we introduce a 2 -step process: First we create a panoramic mosaic from the images. We then align small image regions to the panorama, and apply super resolution resulting in a panoramic image with higher resolution.
\end{abstract}

\section{Panoramic Mosaicing}

Video sequences of a scene can be compactly represented in a single image using panoramic mosaicing. The images are projected onto a common manifold $[7,6,5,3,8]$ to create a single panoramic image. See Fig. 1.

Two methods to construct a mosaic are:

(i) combining the aligned images by a median, average etc.

(ii) each region in the mosaic is taken only from one of the overlapping images.

Method (i) requires accurate alignment over the entire image area, otherwise the resulting mosaic will be blurred. Method (ii) requires alignment only along the seams, but blending is necessary to conceal gain differences.

\section{Super Resolution}

Super-resolution algorithms take advantage of overlapping regions which appear in multiple frames. Using the image displacements, which should be computed with a subpixel accuracy, the multiple overlapping frames provide a dense sampling, and thus enable restoring high frequencies and improve resolution. There several methods to incorporate several overlapping images into a single high-resolution image. See $[2,4,1]$ for further details.

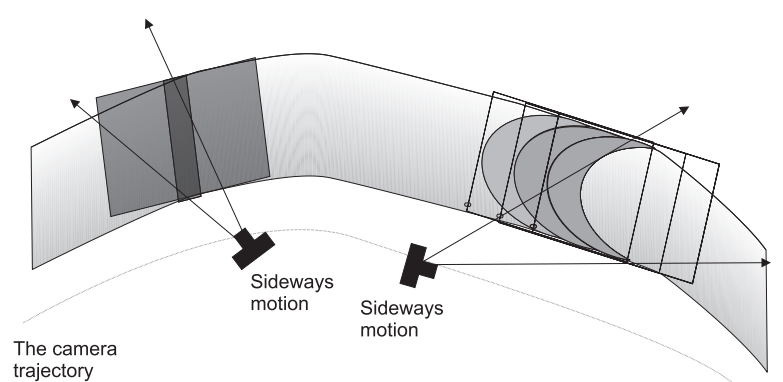

Figure 1. Manifold mosaicing is the process of aligning strips along the seams so the optical flow between each two strips is parallel. The result is general non-rectangular strips.

\section{Combining Super Resolution and Mosaicing}

Manifold mosaicing emphasizes image alignment in the seams between strips, whereas for super resolution algorithms alignment accuracy over the entire overlapping region is very important: image resolution can be improved only when an accurate alignment can be computed. Thus the image alignment for mosaicing may not be accurate enough for super resolution. We present a method for combining super resolution into manifold mosaicing even in cases where the alignment used for mosaicing may not be accurate over the overlapping regions.

The proposed algorithm starts with creating the panorama using generalized strips $[6,5]$. As the first step in the super resolution algorithm, the panorama is expanded to the desired resolution using some image interpolation method (for example a bilinear interpolation). The resolution is enhanced using the following steps for each strip in the panorama:

1. All images containing this strip are collected, and the location of this strip in these images is computed using the parameters found at the initial image alignment step.

2. All overlapping frames are aligned to the strip in the panorama. This alignment is computed only for the strip and a small neighborhood, and may use a different align- 
a)
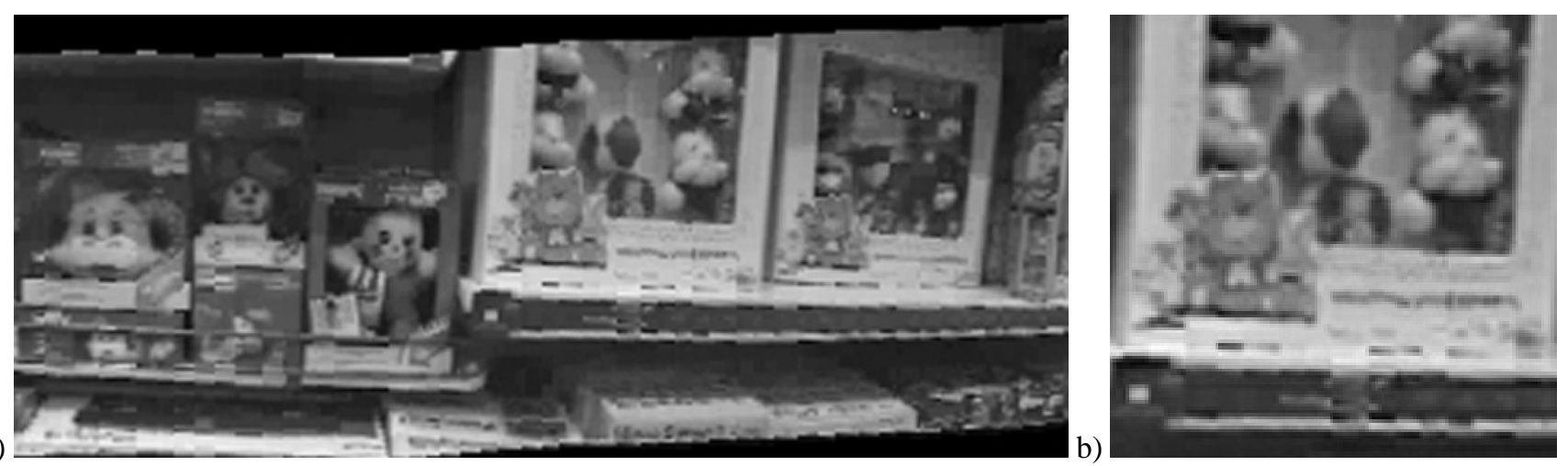

c)
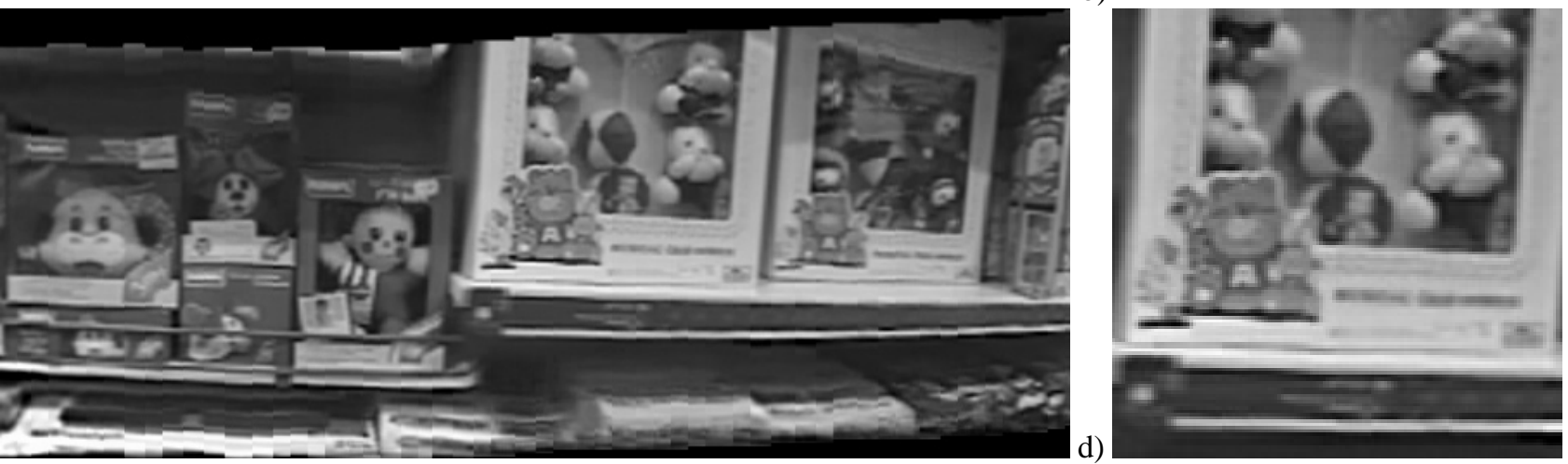

\section{Figure 2. A Panoramic mosaic with a detailed window before super resolution $(a, b)$ and after super resolution $(c, d)$. The camera motion included rotation and translation.}

ment model than used for mosaicing. For example, even when the mosaic algorithm uses a rigid alignment with only image translation and image rotation (3 parameters), the alignment in this step may use an affine model (6 parameters) or a homography (8 parameters). Since only a strip is aligned, and not the entire image, and since a more accurate model may be used, this alignment will be more accurate than the alignment used for mosaicing.

3. From all images aligned to the strip, images with poor quality or poor alignment are removed. Image quality may include sharpness, noise, dynamic range, and other quality criteria.

4. A super resolution algorithm [2] is applied to the strip using the selected images. The strip in the panorama is enhanced without changing the geometry of the panorama. The seams in the original panorama, the result of sampling artifacts, noise and gain differences, also disappear. An example is shown in Fig. 2

\section{Summary}

Super resolution and panoramic mosaicing can be combined by using the enlarged panoramic image as the reference for registration. The resolution of a panoramic image is enhanced without distorting its geometric structure. This process also smoothes the seams without degradation in the image quality.

\section{References}

[1] M. Elad and A. Feuer. Restoration of single super-resolution image from several blurred, noisy and down-sampled measured images. In IEEE Trans. on Image Processing, pages 1646-1658, December 1997.

[2] M. Irani and S. Peleg. Improving resolution by image registration. In CVGIP: Graphical Models and Image Processing, pages 231-239, May 1991.

[3] R. Kumar, P. Anandan, M. Irani, J. Bergen, and K. Hanna. Representation of scenes from collections of images. In IEEE Workshop on Representations of Visual Scenes, pages 10-17, 1995.

[4] A. Patti, M. Sezan, and M. Tekalp. Superresolution video reconstruction with arbitrary sampling lattices and nonzero aperture time. In IEEE Trans. on Image Processing, pages 1064-1078, August 1997.

[5] S. Peleg and J. Herman. Panoramic mosaics by manifold projection. In IEEE Conf. Computer Vision and Pattern Recognition, pages 338-343, June 1997.

[6] B. Rousso, S. Peleg, and I. Finci. Mosaicing with generalized strips. In DARPA Image Understanding Workshop, pages 255-260, May 1997.

[7] B. Rousso, S. Peleg, I. Finci, and A. Rav-Acha. Universal mosaicing using pipe projection. In International Conf. on Computer Vision, pages 945-952, January 1998.

[8] R. Szeliski. Video mosaics for virtual environments. IEEE Computer Graphics and Applications, 16:22-30, March 1996. 